\title{
Methodology for creating 3D paper unfolded models with complex geometry using open-source software and resources with free personal and commercial license
}

\author{
Kremena Tsankova Cankova ${ }^{1}$, Tihomir Atanassov Dovramadjiev ${ }^{1}$ and Ginka Velikova Jecheva ${ }^{1}$ \\ 1-Technical University of Varna, Department Industrial Design, 9010, 1 Studentska Street, Varna, Bulgaria \\ Corresponding author contact: kremenacankovalabv.bg
}

\begin{abstract}
The creation of 3D paper unfolded models with complex geometry requires precision in the design process. Achieving this is through the use of accessible resources and modern technological means which possess the necessary technical and functional capabilities. The successful realization of the models is through the use of a structured methodology containing design stages that are defined in the exact sequence. The present study aims to create the unfoldings of complex three-dimensional models by covering the stages of providing accessible resurses with a personal and commercial license that are transformed in sequence until the readymade variants.
\end{abstract}

Keywords: Inkscape, Blender, 3D, paper, models

\section{Introduction}

Blender software automates the realization of the unfoldings of three-dimensional polygonal mesh models (Cankova K., Dovramadjiev T. and Jecheva G., 2017). In order to do this, it is necessary to have certain conditions such as: a properly structured mesh of the three-dimensional model, the geometric element and the number of polygons. These conditions are interrelated and are subject to research in this article. With the implementation of several sample models with complex 3D geometry, it will be possible to gain a deep understanding of the process of creating unfolding of the sample patterns. For the making of the samples, a methodology has been used that is optimized in a way that allows designers to have all available resources and technical criteria for the realization of triple polygonal mesh model unfolds, which are often used to make real paper models for the needs of art design (Molina, i-link visited 2018), sculpture, architecture(Xian-Ying, Chao-Hui, Shi-Sheng, Tao \& Shi-Min, 2010), jewellery (Halbrook, i-link visited 2018), etc.

Different techniques and resources are used in the design process. Referring to specific patterns such as symbols, letters, ornaments, figures of people, animals, buildings, vehicles, etc., it is a good opportunity to use ready-made vector models. Such resource is http://www.fontspace.com/, where free fonts are available with a personal and / or commercial license. Some specific fonts have ready-made vector images that can be directly used in 3D geometry modelling, while respecting a particular process path of transformation from the vector image to the raster and vector again in order to create a model footprint. If a raster image obtained from another resource is used, it is converted directly from a raster to a vector (Sonal D. B., Jadhav P. M., 2016). The saved (scalable) file in Scalable Vector Graphics (*.svg) is then extruded and used in the process a Boolean operation that builds the finished three-dimensional geometry of the final model. The resulting three-dimensional model is completely ready, and an automated process (using the Blender add-on Export: Paper Model) creates a unfolding that is saved in *.pdf file format (Balbio V., Rachinskiy M., Topi T., Meta-Androcto, i-link visited 2018).

This study examines in detail the features of the used software products (Alecu F., 2010, Blender software, i-link visited 2018, Chronister J., 2009 \& 2011, Chopine A., 2011, Inkscape 
software, i-link visited 2018, Dovramadjiev T., 2015, Wickes, 2009; Prahov, 2009), their specific functional qualities and the right approach for the production of unfoldings of three-dimensional models for making them from paper.

\section{Materials and Methods}

Given the many features accompanying the development of unfoldings of 3D models with complex geometry, the construction of an optimal methodology is a necessity.

In Fig. 1, there is shown a method for creating a three-dimensional pattern unfolding comprising the following steps:

1. Providing vector and / or raster resource of symbols, letters, ornaments, figures of people, animals, buildings, vehicles, etc..

2. Creating a vector footprint (negative image of the image) by tracing the image in the Inkscape open-source special software.

3. Importing the svg (Scalable Vector Graphics) file into a Blender environment (open-sourse software running on polygonal mesh models).

4. Extrusion of the vector into a volumetric body.

5. Using Boolean operations to create (cast) the finished 3D model.

6. Automated creation of unfolds.

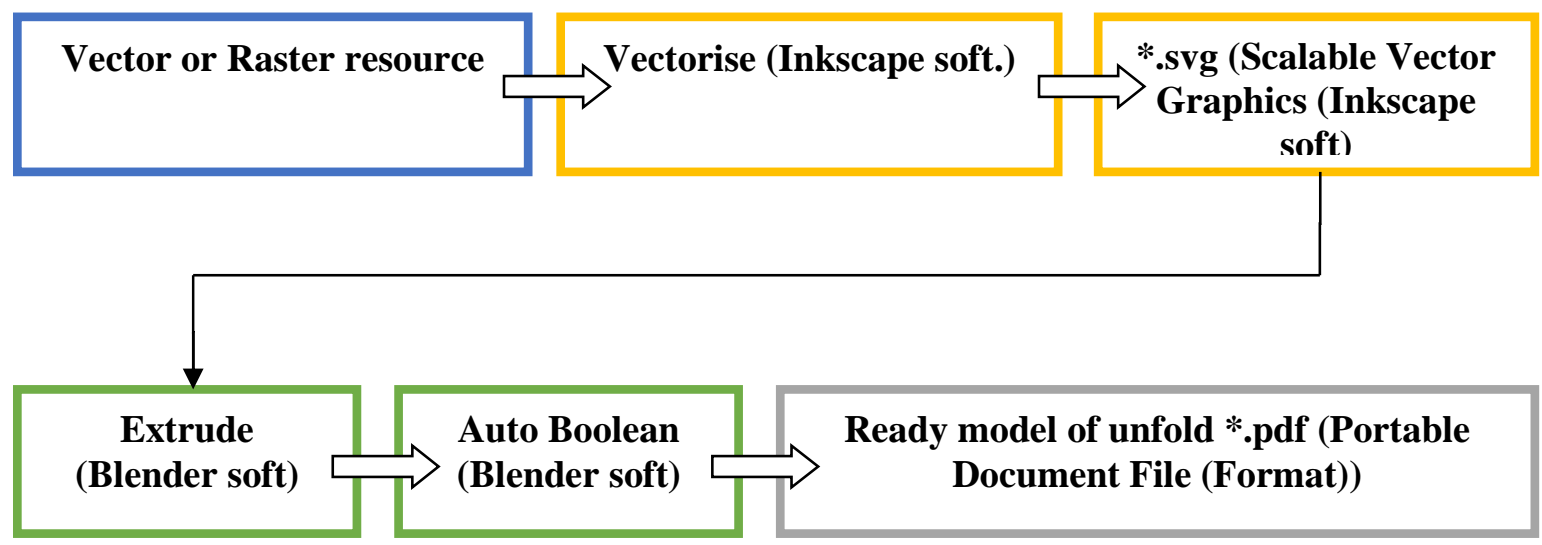

Fig. 1. Method to create infoldings of complex three-dimensional models.

The efficient use of the methodology is applied in the development of an exemplary vector model - an ornament that is provided through http://www.fontspace.com/. The resource has a free and personal and commercial license (Fig. 2). 


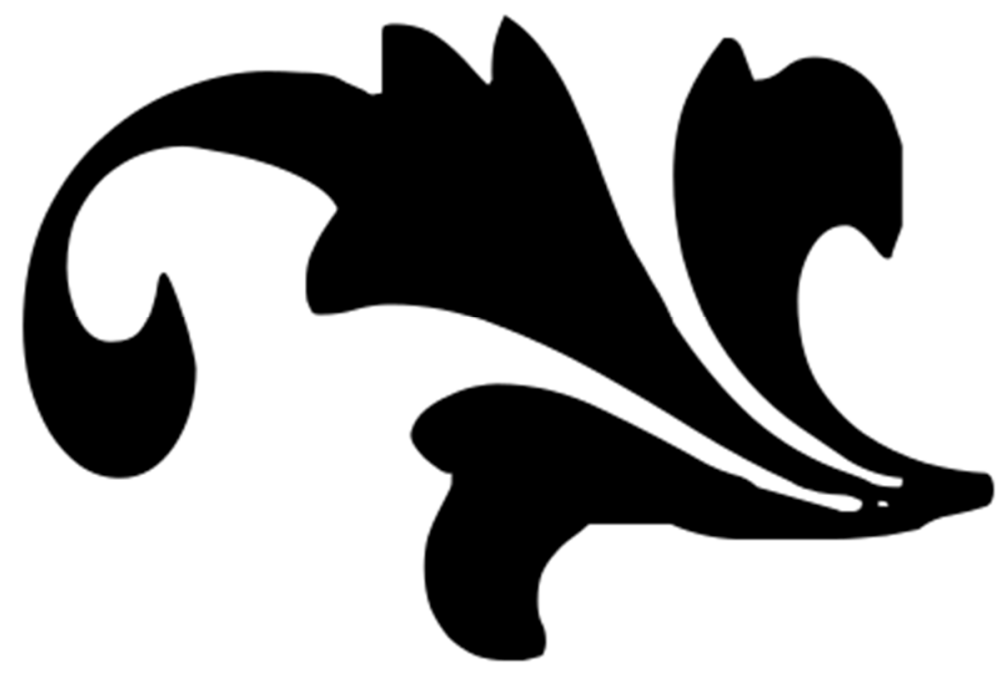

Fig. 2. Providng the resource ("P" key). WWFloral Corner font.Licensed as: Freeware Personal Use \& Commercial Use. http://www.fontspace.com/windwalker64/wwfloralcorner.

The font "WWFloralCorner.ttf" (WWFloralCorner font) is installed in the "Fonts" folder of OS Windows and opens in the open-source Inkscape. A white background needed to create the print is added to the vector ornament. The model is captured in an image. Creating a vector footprint of the resulting image using Path> Trace Bitmap (Shift + Alt + B). In the "Trace Bitmap" window is marked "Invert image", and it is desirable to use "Live Preview" (Fig 3).

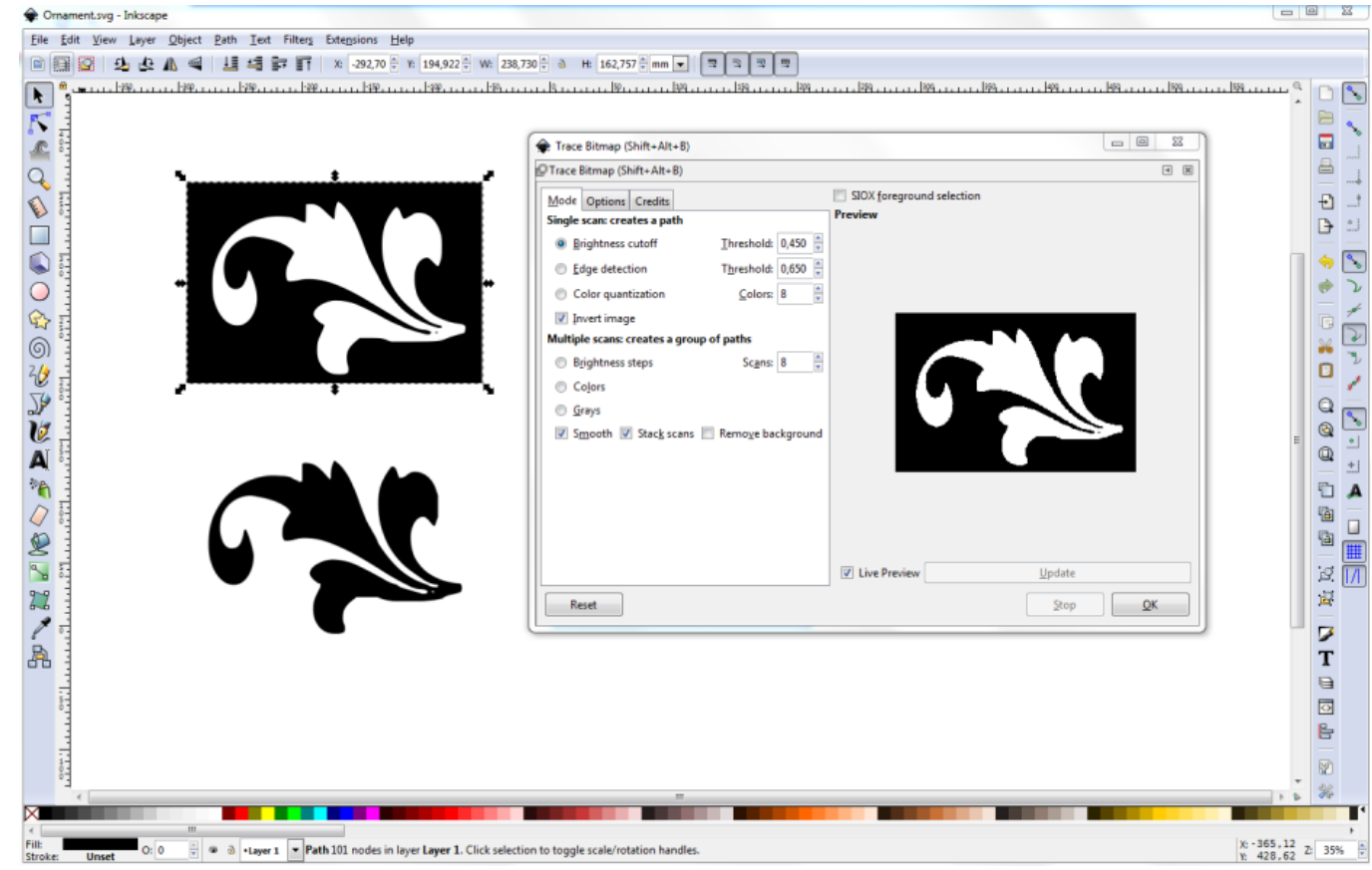

Fig. 3. Trace Bitmap (Inkscape software).

Scalable Vector Graphics file-ornament is imported into Blender software via "File"> "Import" (Fig. 4). The resulting object is rotated, positioned and extruded at $0.15 \mathrm{~m}$ (Fig. 5) 


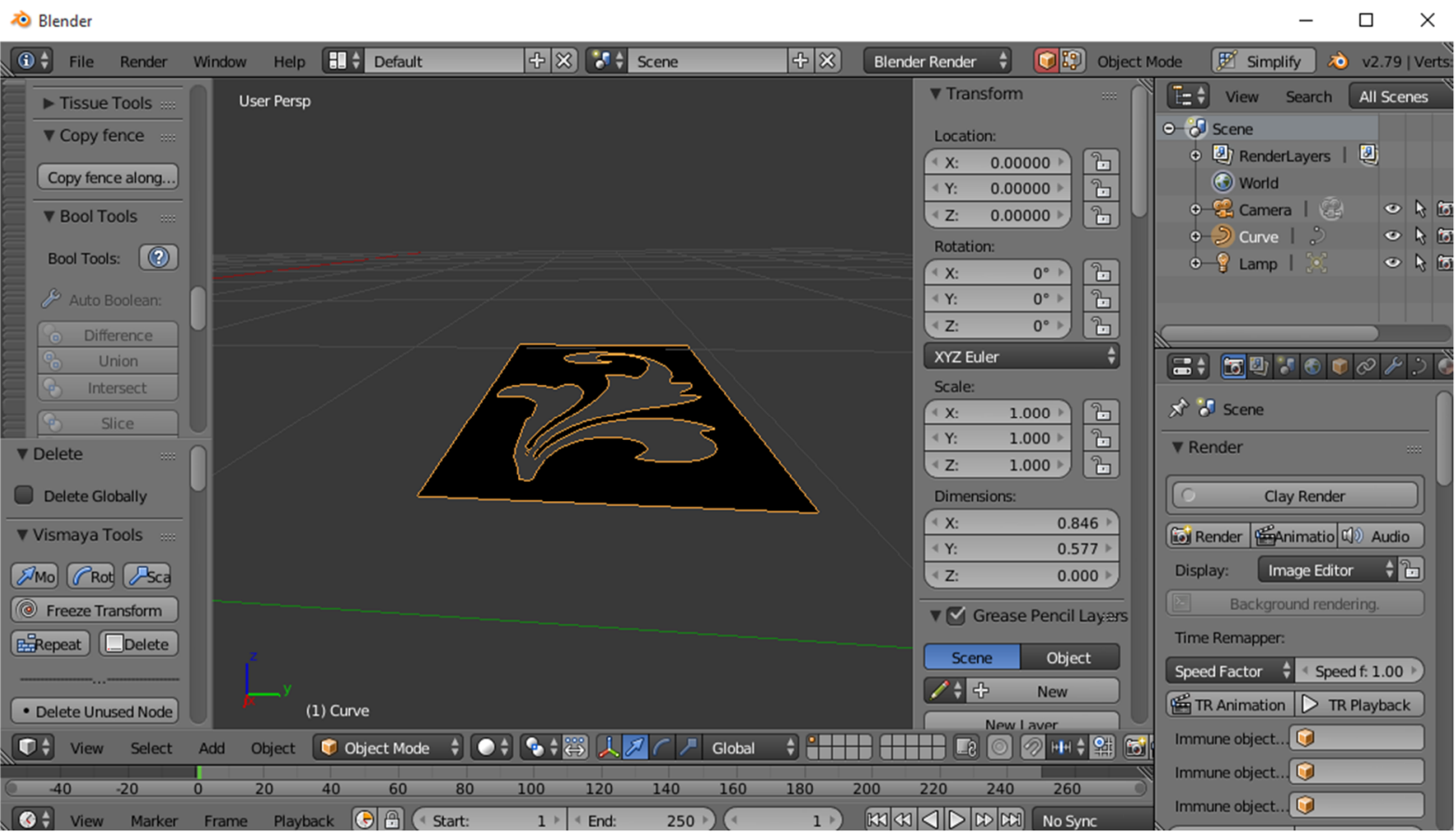

Fig. 4. Scalable Vector Graphicsfile-ornament is imported into Blender software.

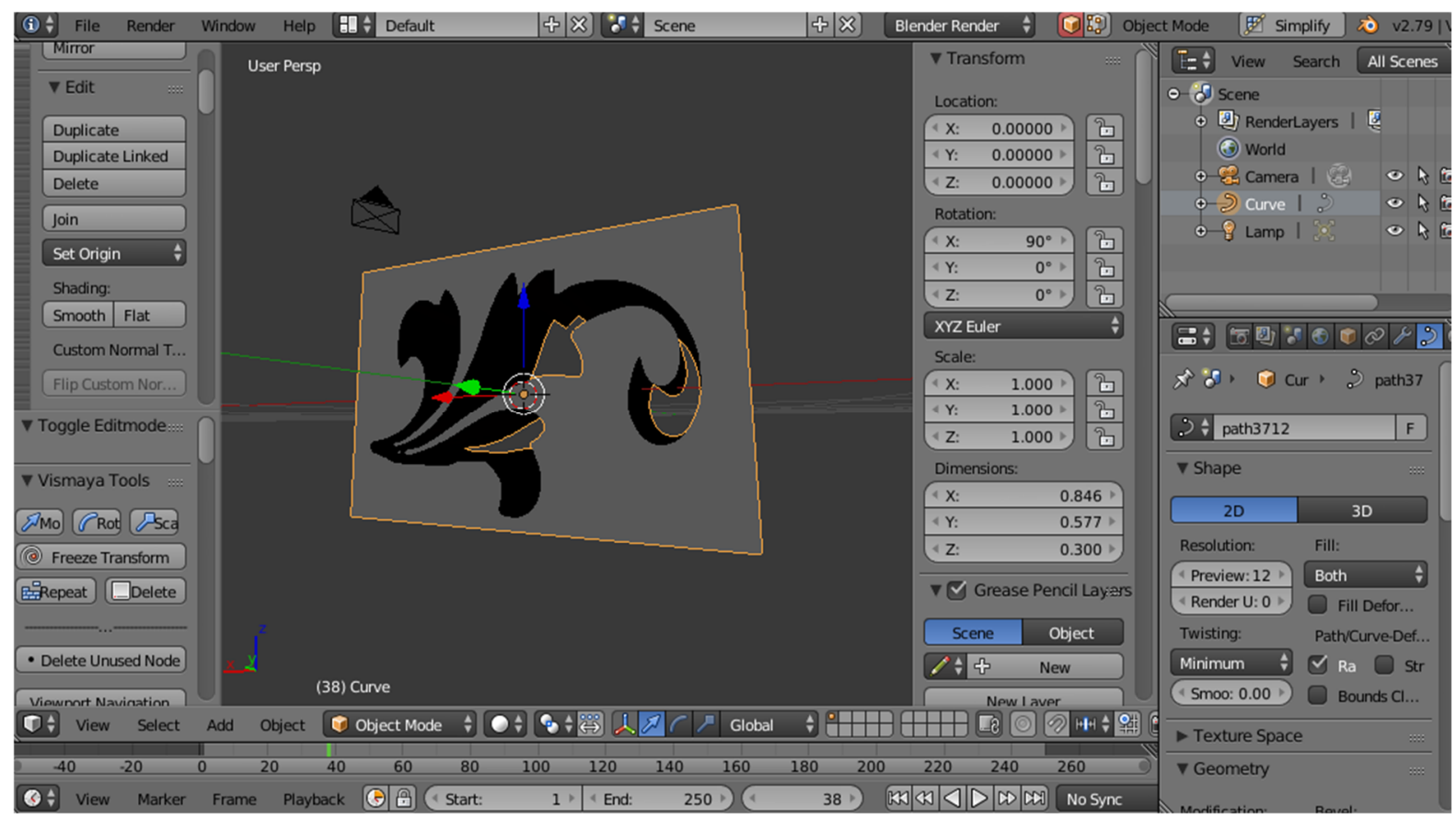

Fig. 5. The object: rotated, positioned and extruded at $0.15 \mathrm{~m}$.

Using Add> Mesh> Cube, a new 3D object is created - primitive cube. It is transformed and positioned in the middle area of the casting mold (Fig. 6). The two objects are sequentially selected for the imported object and the "cube" and the Boolean operation is performed Brush Boolean / Difference (Fig. 7; Bool Tool Addon - Author(s) Vitor Balbio, Mikhail Rachinskiy, Tynka Topi, Meta-Androcto). 


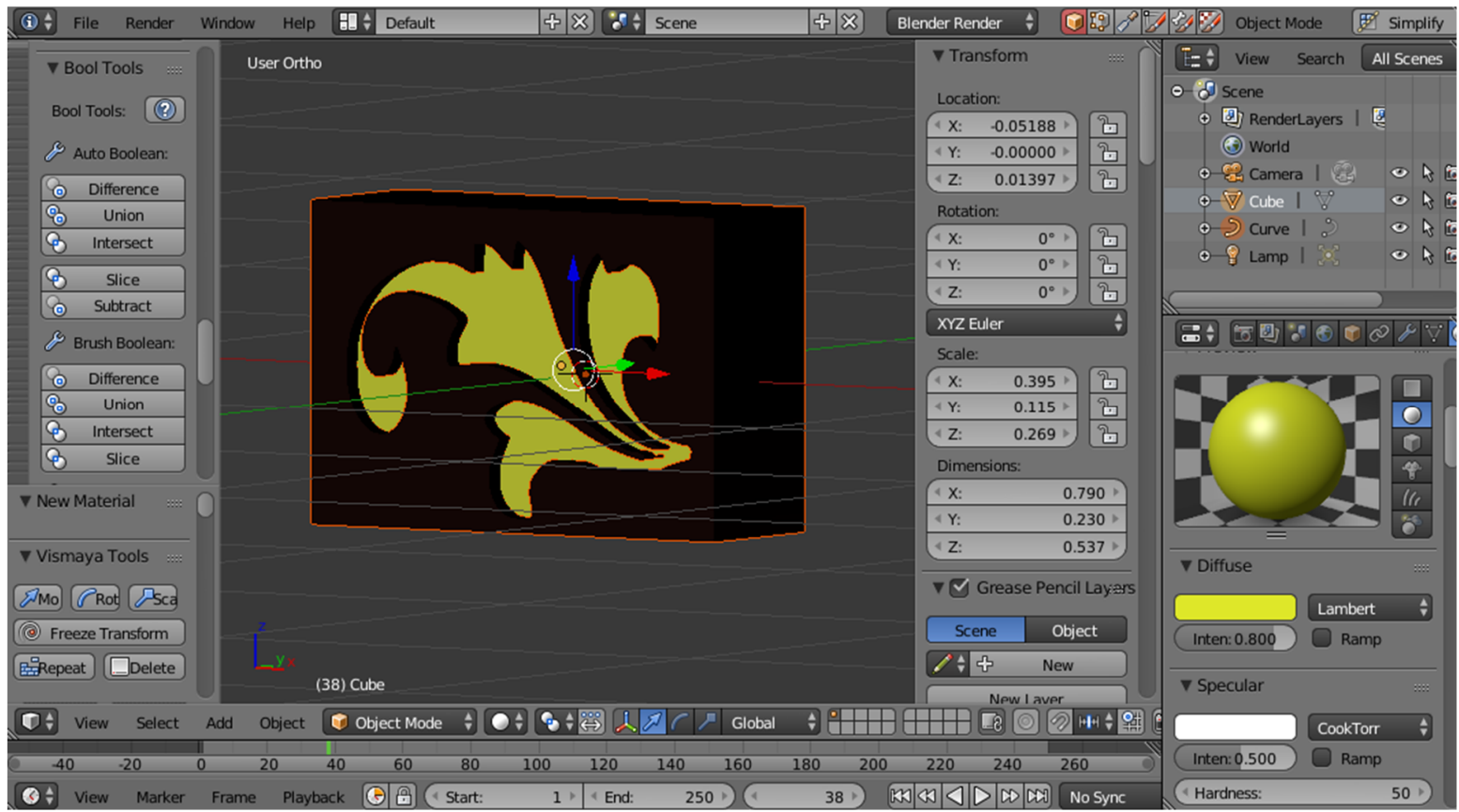

Fig. 6. Preparation for casting of the form in a computer environment.

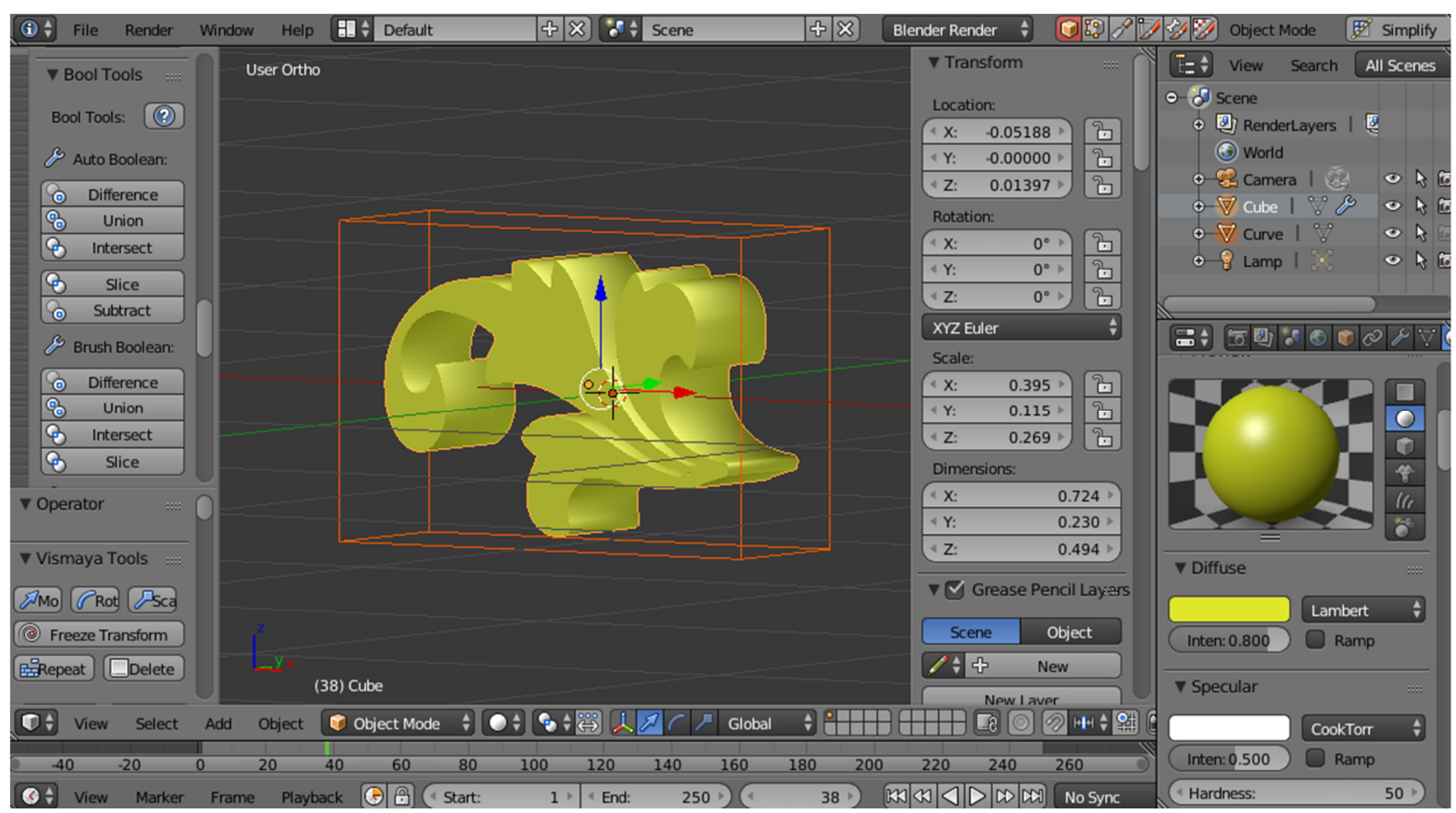

Fig. 7. The resulting shape of the pattern - ornament following a Boolean operation.

Upon receipt of the form, it goes to the construction of the polygonal mesh through the Object $>$ Convert to (Alt C) > Mesh from Curve/Meta/Surf/Text (Fig. 8). 


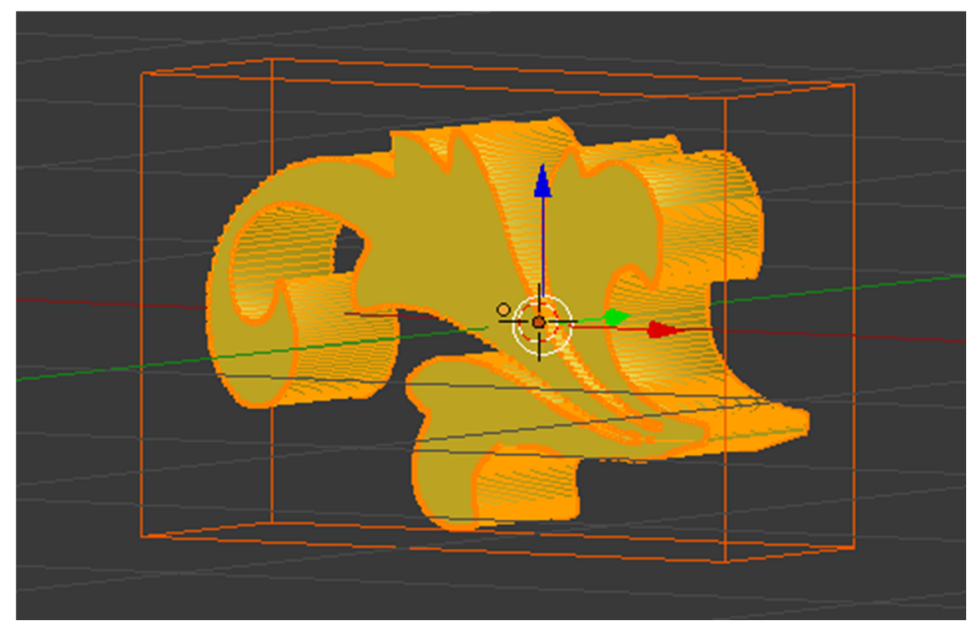

Fig. 8. The resulting ready three-dimensional pattern - ornament. In Edit Mode, the model's mesh structure is polygonally displayed.

\section{Results}

Using the Blender Addon: Export Paper Model, the unfolding of the model-ornament is automatically made. The result is shown in Fig. 9 (a). In Fig. 9 (b) and (c) there are other complex models shown that are made using the same methodology.
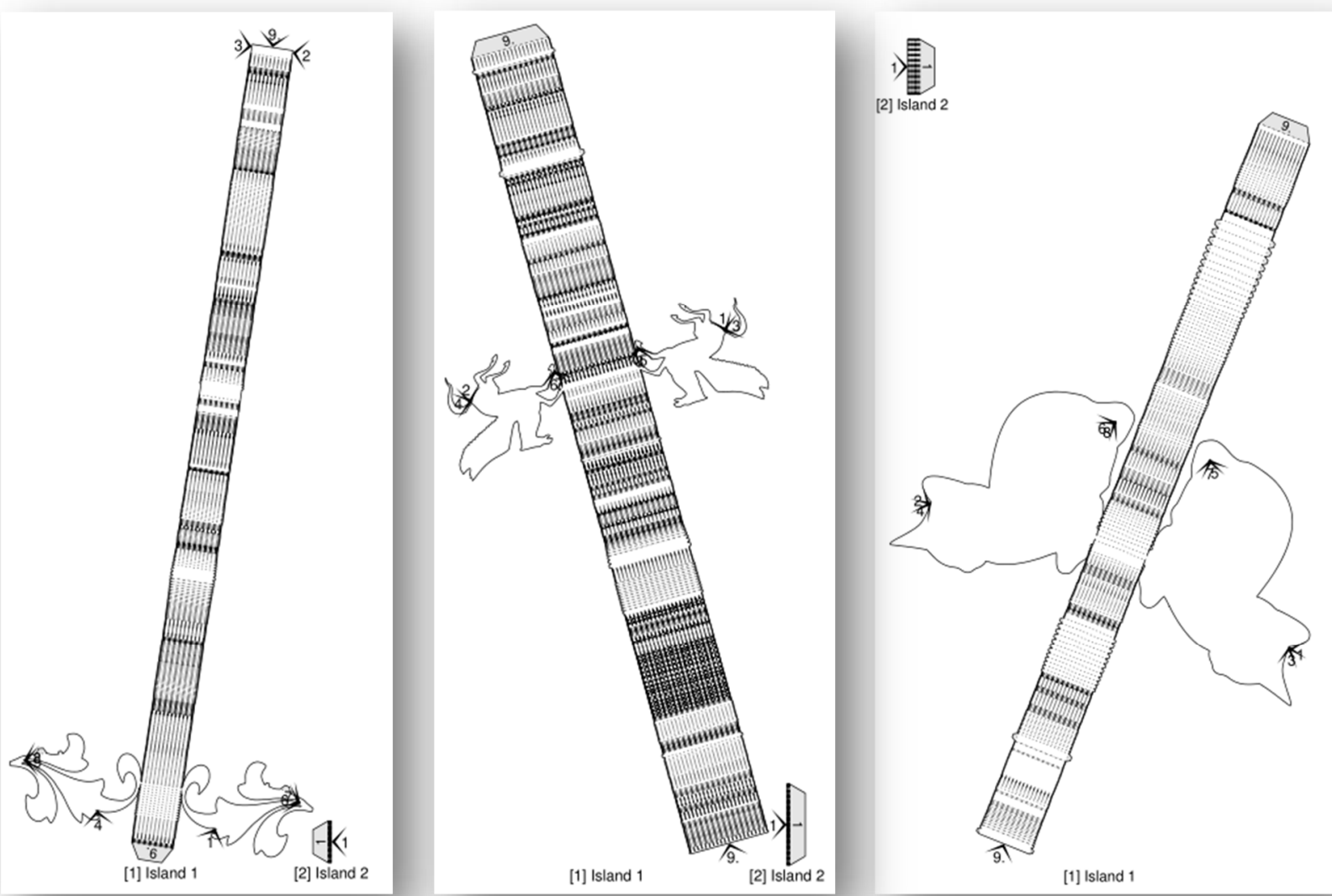

Fig. 9. The resulting unfolding (a) ornament - sample model (b) pegasus: WWFantasy font http://www. fontspace.com/windwalker64/wwfantasy(c) cat: Cats vs Dogs LT font http://www.fontspace.com/ladytimeless/cats-vs-dogs-lt 


\section{Conclusion}

In the present study, a model of a three-dimensional polygon mesh object was made using a newly developed methodology, providing the necessary conditions for successful realization. The here shown results of the made unfolds and other models with complex geometry characterize the defined technological path as a fully functional. Based on this, as well as the availability of the free license and open-source technology tools, it is concluded that the constructed methodology provides suitable conditions for the realization of paper models by automating the process. This leads to an increase in the accuracy and quality of the obtained models.

\section{References}

Alecu F. (2010). Blender Institute - the Institute for Open 3D Projects, Open Source Science Journal Vol. 2, No. 1, Economic Informatics Department, ASE Bucharest, Romania, p. 36 - 45.

Chopine A. (2011). 3D Art Essentials The Fundamentals of 3D Modeling, Texturing, and Animation, Elsevier, ISBN: 978-0-240-81471-1, USA, p. 249 - 252.

Cankova K., Dovramadjiev T. and Jecheva G. (2017). Computer parametric designing in Blender software for creating 3D paper models. ANNUAL JOURNALOF TECHNICAL UNIVERSITY OF VARNA. Vol.1 Issue 1, p. $77-84$.

Chronister J. (2009). Blender 3D Basics 3rd Edition, Creative commons attribution-Non Commercialshare alike 3.0 Unported License, p.146.

Chronister J. (2011). Blender Basics 2.6 (4-rd edition). Cdschools eBook, p. 178.

Dovramadjiev T. (2015). "Modern accessible application of the system Blender in 3d design practice". International scientific on-line journal "SCIENCE \& TECHNOLOGIES". Publishing House "Union of Scientists - Stara Zagora", ISSN 1314-4111; p. 10 - 13.

Sonal D. Borhade, P. M. Jadhav (2016). Conversion of Bitmap Image to Vector Image for Image Processing Applications. International Engineering Research Journal (IERJ) Volume 2 Issue 2. ISSN 2395-162; India. p. 663-667.

Straub Raphael and Prautzsch Hartmut (2011). Creating Optimized Cut-Out Sheets for Paper Models from Meshes. Karlsruhe Reports in Informatics 36. Edited by Karlsruhe Institute of Technology, Faculty of Informatics, ISSN 2190-4782.

Wickes D. R. (2009). Foundation Blender Compositing, ISBN-13 (pbk): 978-1-4302-1976-7, USA.

Xian-Ying Li 1 Chao-Hui Shen 1 Shi-Sheng Huang 1 Tao Ju 2 Shi-Min Hu 1(2010). _Automatic Paper Architectures from 3D Models. 1 TNList, Department of Computer Science and Technology, Tsinghua University, Beijing. 2 Department of Computer Science and Engineering, Washington University in St. Louis.

Прахов А. А. (2009). Blender: 3D-моделирование и анимация. СПб.: БХВ, ISBN 978-5-97750393-8, Русия, 272 стр 


\section{Online sources (visited 2018)}

Balbio V., Rachinskiy M., Topi T., Meta-Androcto. BoolTool Add-on. 2.7x License GPL. https://wiki.blender.org/index.php/Extensions:2.6/Py/Scripts/Object/BoolTool

Blender software. About \& License. https://www.blender.org/about/

DominecA. (Emu). Export: Paper Model (.svg) Add-on. Export printable net for paper modeling. License GPL. https://wiki.blender.org/index.php/Extensions:2.6/Py/Scripts/ImportExport/Paper_Model

FontSpace.Licensed as: Freeware / Personal Use / Commercial Use:

- LadyTimeless (2005). Cats vs Dogs LT font.

http://www.fontspace.com/ladytimeless/cats-vs-dogs-lt;

- WindWalker64 (1999).WWFloralCorner font. http://www.fontspace.com/windwalker64/wwfloralcorner;

- WindWalker64 (1999). WWFantasy font. http://www.fontspace.com/windwalker64/wwfantasy.

Inkscape software. About. https://inkscape.org/en/about/overview/

Molina C. Paper \& Digital Artistry. http://carlosnmolina.com

Halbrook K.E. Create 3D paper sculpture jewelry, home decor and gifts. bayoudreamer.com/pdfs/paper_jewelry.pdf 